\title{
Sparse Covariance Fitting Method for Direction of Arrival Estimation of Uncorrelated Wideband Signals
}

\author{
Slavche Pejoski and Venceslav Kafedziski
}

\begin{abstract}
We propose a new direction of arrival estimation method for wideband uncorrelated signals. The wideband signals are first decomposed into narrowband signals. A group sparse Lasso formulation is proposed that jointly fits the powers of the signals using overcomplete dictionaries of the directions of arrival, into the estimated covariance matrices for all narrowband signals. Then, we propose a new formulation that determines the regularization parameters and becomes the group Lasso formulation. Additionally, we propose a modified algorithm for direction of arrival estimation with lower complexity that uses conventional based methods in the preprocessing stage to reduce the number of variables in the optimization task. We compare the performance of the proposed method to the conventional methods for a circular antenna array.

Keywords - COMET, covariance fitting, direction of arrival estimation, group sparse Lasso, wideband signals.
\end{abstract}

\section{INTRODUCTION}

$\mathrm{T}$ HE direction of arrival (DoA) estimation problem is one of the most investigated problems in the antenna array signal processing community. Many solutions have been proposed in the past [1]-[3], but the problem is still relevant. The introduction of conditions for perfect reconstruction in compressed sensing [4] increased the interest for sparse modeling and this is reflected in the DoA estimation problem. Namely, two new approaches that are based on the sparse modeling have been introduced in this field. The first approach directly applies the sparse model to the sampled data [5]-[7] and the second approach applies the sparse model to the covariance matrix of the received signal [8]-[12]. Additionally, new approaches based on time [13], [14] and antenna [15] subsampling have been proposed. Most of these approaches are intended for narrow band signals, but some can be converted to wideband by introducing group

Paper received March 30, 2014; revised September 29, 2014; accepted September 30, 2014. Date of publication November 15, 2014. The associate editor coordinating the review of this manuscript and approving it for publication was Prof. Branimir Reljin.

This paper is a revised and expanded version of the paper presented at the 21th Telecommunications Forum TELFOR 2013.

Slavche Pejoski is with the Faculty of Electrical Engineering and Information Technologies, University Cyril and Methodius, Republic of Macedonia (phone: +38923099112; fax: +38923064262; e-mail: slavchep@feit.ukim.edu.mk).

Venceslav Kafedziski is with the Faculty of Electrical Engineering and Information Technologies, University Cyril and Methodius, Republic of Macedonia (phone: +38923099120; fax: +38923064262; e-mail: kafedzi@feit.ukim.edu.mk). sparsity in the model. In array processing a narrowband signal is usually defined as a signal that meets the condition about the bandwidth to center frequency being less than $1 \%$ [16], [17]. Here we extend the narrowband model from [10], [11] to a wideband model and derive a new wideband direction of arrival estimation method which is the novelty of this paper. The received wideband signal is first decomposed into a set of independent narrowband signals using Discrete Fourier Transform (DFT) ([2], [3]) or narrowband filters ([6]). For each of the different narrowband signals the covariance matrix is calculated and a sparse narrowband model based on individual sparsity is created. Then, the sparse narrowband models are jointly treated through a group sparsity constraint. Instead of the usual substitution of the individual sparsity constraint with a group sparsity constraint, we keep both constraints and obtain a group sparse Lasso problem [18], [19]. We choose the individual sparsity regularization parameter as the one that is optimal for the infinite number of obtained snapshots. This parameter choice allows us to reformulate the problem as a purely group Lasso problem and make a connection to the Covariance Matching Estimation Technique (COMET) framework from [20] to determine the second regularization parameter for the group sparsity, based on the noise in the system. We compare the performance of the system with conventional methods for wideband direction of arrival estimation. Additionally, similarly as in [21], we propose a modified algorithm that uses conventional methods for preprocessing and makes the complexity of the algorithm proportional to the number of detected directions of arrival in the preprocessing phase.

The rest of this paper is organized as follows. In the second section we give a theoretical explanation of the proposed method and the reduced complexity algorithm. In section III we present the simulation results showing the performance of our proposed method. Section IV concludes the paper.

\section{DESCRIPTION OF THE METHOD}

In our considered system the antenna array has $P$ antennas (sensors) that receive $K$ uncorrelated wideband signals arriving from different directions $\theta_{k}$. The output of every antenna is corrupted with additive white Gaussian noise. The antenna outputs can be directly sampled as in acoustic systems or first downconverted and then sampled as in communication systems, and then processed using 
DFT with a sufficient number of frequency bins as in [2], [3] or narrowband filters followed by additional conversion to baseband as in [6]. Thus, we obtain a set of $M$ baseband signals representing the $M$ narrowband signals at different frequencies $f_{m}$ (corresponding to the modulated signal):

$$
\mathbf{y}_{m}(n)=\mathbf{A}_{m}\left(f_{m}\right) \mathbf{x}_{m}(n)+\mathbf{v}_{m}(n)
$$

where $\quad \mathbf{y}_{m}(n)=\left[y_{m 1}(n) y_{m 2}(n) \ldots y_{m P}(n)\right]^{T}$ is a vector containing the $n^{\text {th }}$ snapshot of the baseband signals representing the received narrowband baseband signals at frequency $f_{m}$ with respect to the reference point in space for each antenna and $m=1,2, \ldots, M$. $\mathbf{x}_{m}(n)=\left[x_{m 1}(n) x_{m 2}(n) \ldots x_{m K}(n)\right]^{T}$ is a zero mean circular complex Gaussian random vector containing the $n^{\text {th }}$ snapshot of the baseband signals representing the narrowband signals at frequency $f_{m}$ that arrive from the different directions. $\mathbf{v}_{m}=\left[v_{m 1}(n) v_{m 2}(n) \ldots v_{m P}(n)\right]^{T}$ is a zero mean circular complex Gaussian random vector containing the $n^{\text {th }}$ snapshot of the baseband representation of the noise in the $m^{\text {th }}$ narrowband channel at each antenna. $\mathbf{A}_{m}\left(f_{m}\right)=\left[\mathbf{a}\left(\theta_{1}, f_{m}\right) \mathbf{a}\left(\theta_{2}, f_{m}\right) \ldots \mathbf{a}\left(\theta_{K}, f_{m}\right)\right], \quad$ where $\mathbf{a}\left(\theta_{k}, f_{m}\right)$ represents the steering vector for the $k^{\text {th }}$ signal at frequency $f_{m}$. For a circular antenna array with a reference point in its center, $\mathbf{a}\left(\theta_{k}, f_{m}\right)=$ $\left[e^{-j \frac{2 \pi}{c} \rho f_{m} \cos \left(\theta_{k}\right)} e^{-j \frac{2 \pi}{c} \rho f_{m} \cos \left(\theta_{k}+\frac{2 \pi p}{P}\right)} \ldots e^{-j \frac{2 \pi}{c} \rho f_{m} \cos \left(\theta_{k}+\frac{2 \pi(P-1)}{P}\right)}\right]^{T}$, where $\rho$ is the radius of the array, $c$ is the speed of waves, the leftmost antenna corresponds to the first entry and the antennas are counted counter-clockwise. The utilization of circular array has some advantages over the usually considered uniform linear arrays, such as more compact design and the ability for spatial discrimination over $360^{\circ}$ of the azimuth range [22]. Additionally, circular antenna arrays have almost angle independent behavior regarding the conditions for distinguishing wideband from narrowband signals.

For the $m^{\text {th }}$ narrowband channel, we use the notation $\mathbf{y}_{m}=\left[\mathbf{y}_{m}(1) \mathbf{y}_{m}(2) \ldots \mathbf{y}_{m}(N)\right] \quad$ and $\quad \mathbf{x}_{m}=\left[\mathbf{x}_{m}(1) \mathbf{x}_{m}(2) \ldots\right.$ $\left.\mathbf{x}_{m}(N)\right]$ where $N$ is the number of snapshots and create the estimate of the covariance matrix of the $m^{\text {th }}$ narrowband signal:

$$
\hat{\mathbf{R}}_{m}=\frac{1}{N} \mathbf{y}_{m} \mathbf{y}_{m}^{H}
$$

where $H$ denotes the conjugate transpose. For clarity, we first explain the processing in the conventional wideband methods. In [3] the matrices of all $M$ signals are resteered using linear transform matrices to a central frequency and then the new matrix is processed using conventional methods, such as MUSIC, on the new matrix. Such methods are known as coherent methods. Another conventional method [2] calculates the spatial spectra of all $M$ signals and combines them to obtain a new spectrum that is used to estimate the directions of arrival (DoAs). This method belongs to the noncoherent group of wideband DoA methods. Now we turn our attention to the sparse covariance fitting methods.

The narrowband sparse covariance fitting methods are based on the true covariance matrix of (1):

$$
\begin{aligned}
\mathbf{R}_{m} & =E\left[\mathbf{y}_{m}(n) \mathbf{y}_{m}(n)^{H}\right] \\
& =\mathbf{A}_{m}\left(f_{m}\right) \mathbf{R}_{x m} \mathbf{A}_{m}\left(f_{m}\right)^{H}+\sigma_{v m}^{2} I_{P}
\end{aligned}
$$

where $\sigma_{v m}^{2}$ is the variance of the noise in the $m^{\text {th }}$ equivalent baseband channel. We assume that the noise variance is equal for all $m$ and in the rest of the paper we simply use $\sigma_{v}^{2}$ for the noise variance. An overcomplete model where the DoA space is divided into $L$ possible directions positioned on a specific grid, can be used to represent the covariance matrix in (3):

$$
\begin{aligned}
\mathbf{R}_{m} & =E\left[\mathbf{y}_{m}(n) \mathbf{y}_{m}(n)^{H}\right] \\
& =\mathbf{A}_{\mathbf{1} m}\left(f_{m}\right) \mathbf{R}_{\mathbf{1}_{x m}} \mathbf{A}_{\mathbf{1}_{m}}\left(f_{m}\right)^{H}+\sigma_{v}^{2} I_{P}
\end{aligned}
$$

where $\mathbf{A}_{1 m}\left(f_{m}\right)=\left[\mathbf{a}\left(\tilde{\theta}_{1}, f_{m}\right) \mathbf{a}\left(\tilde{\theta}_{2}, f_{m}\right) \ldots \mathbf{a}\left(\tilde{\theta}_{L}, f_{m}\right)\right]$ is the steering matrix of all $L$ possible directions, where $L>P, \mathbf{R}_{\mathbf{1}_{x m}}$ represents the correlation matrix of all possible DoAs and is a sparse matrix. When the signals are uncorrelated $\quad \mathbf{R}_{\mathbf{1}_{x m}}=\operatorname{diag}\left\{s_{m 1}, s_{m 2}, \ldots, s_{m L}\right\} \quad$ is a sparse diagonal matrix and $s_{m l}$ is the power of the signal arriving from direction $\tilde{\theta}_{l}$ measured at the $m^{\text {th }}$ narrowband channel, so $s_{m l}$ are real positive numbers. Using this simplification, (2) can be reformulated [10], [11] as:

$$
\hat{\mathbf{r}}_{m}=\operatorname{vec}\left(\hat{\mathbf{R}}_{m}\right)=\mathbf{A}_{\mathbf{2}} \mathbf{s}_{m}+\mathbf{E}
$$

where $\mathbf{s}_{m}=\left[\begin{array}{llll}s_{m 1} & s_{m 2} & \ldots & s_{m L}\end{array}\right]^{T}$ and $\mathbf{E}$ represents the error signal due to noise and estimation error. $\mathbf{A}_{\mathbf{2} m}=$ $\left[\operatorname{vec}\left(\mathbf{a}\left(\tilde{\theta}_{1}, f_{m}\right) \mathbf{a}\left(\tilde{\theta}_{1}, f_{m}\right)^{H}\right) \ldots \operatorname{vec}\left(\mathbf{a}\left(\widetilde{\theta}_{L}, f_{m}\right) \mathbf{a}\left(\tilde{\theta}_{L}, f_{m}\right)^{H}\right)\right], \quad$ where vec denotes vectorization. An efficient estimator for this model according to [10] is the estimator based on the constrained Lasso formulation:

$$
\begin{aligned}
& \min _{\mathbf{s}_{m}}\left\|\hat{\mathbf{r}}_{m}-\mathbf{A}_{2 m} \mathbf{s}_{m}\right\|_{2}^{2}+\beta\left\|\mathbf{s}_{m}\right\|_{1} \\
& \text { s.t. } s_{m l} \geq 0 \text { for } l=1, \ldots, L
\end{aligned}
$$

where $\|\mathbf{x}\|_{1}=\sum_{l=1}^{L}\left|x_{l}\right|$ is the $l_{1}$ norm of $\mathbf{x}$. The optimal value of the regularization parameter $\beta$ for the case of an infinite number of snapshots is $\beta=2 P \sigma_{v}^{2}$ [10].

Now we can construct the wideband direction of arrival method by combining the estimation problems of all $M$ narrowband signals. The different $M$ narrowband signals can be observed as a Multiple Measurement Vector (MMV) fitting model. Since all $M$ narrowband signals are equally important it is expected that they simultaneously have high values of the power $s_{m l}$ for the same directions of arrival from the set $\tilde{\theta}_{l} l=1, \ldots, L$. In this situation it is logical to combine the $M$ optimization problems into a single problem and replace the $l_{1}$ norm in (6) with $l_{2,1}$ norm enforcing group sparsity. Instead, we combine the $M$ narrowband problems by adding the group enforcing norm but keep the $l_{1}$ norm to obtain a group sparse Lasso [18], [19] formulation of the wideband 
DoA estimation problem:

$$
\begin{aligned}
& \underset{\mathbf{s}_{1}, \ldots \mathbf{s}_{M}}{\min } \sum_{m=1}^{M}\left\|\hat{\mathbf{r}}_{m}-\mathbf{A}_{2} \mathbf{s}_{m}\right\|_{2}^{2}+\beta \sum_{m=1}^{M}\left\|\mathbf{s}_{m}\right\|_{1} \\
& \quad+\lambda \Gamma\left(\mathbf{s}_{1}, \ldots, \mathbf{s}_{M}\right) \\
& \text { s.t. } s_{m l} \geq 0 \text { for } m=1, \ldots, M l=1, \ldots, L
\end{aligned}
$$

where $\Gamma\left(\mathbf{s}_{1}, \ldots, \mathbf{s}_{M}\right)$ represents the group sparsity norm i.e. $\Gamma\left(\mathbf{s}_{1}, \ldots, \mathbf{s}_{M}\right)=\sum_{l=1}^{L} \sqrt{s_{1 l}^{2}+s_{2 l}^{2}+\ldots+s_{M l}^{2}}$. Finding suitable values of the regularization parameters and giving them a physical meaning is a challenging task. We propose using the $\beta$ value for the case of an infinite number of snapshots. Additionally, we use the fact that $s_{m l}$ are positive real numbers, so $\left|s_{m l}\right|_{1}=s_{m l}$, and (7) becomes:

$$
\begin{aligned}
\min _{\mathbf{s}_{1}, \ldots \mathbf{s}_{M}} & \sum_{m=1}^{M}\left(\left\|\hat{\mathbf{r}}_{m}-\mathbf{A}_{\mathbf{2}_{m}} \mathbf{s}_{m}\right\|_{2}^{2}+2 P \sigma_{v}^{2} \mathbf{1}^{T} \mathbf{s}_{m}\right) \\
& +\lambda \Gamma\left(\mathbf{s}_{1}, \ldots, \mathbf{s}_{M}\right) \\
\text { s.t. } s_{m l} & \geq 0 \text { for } m=1, \ldots, M l=1, \ldots, L
\end{aligned}
$$

where $\mathbf{1}=[11 \ldots 1]^{T}$ is a column vector of ones with length equal to $L$. With this choice of $\beta$, it is shown in the Appendix that (8) is equivalent to:

$$
\begin{aligned}
\min _{\mathbf{s}_{1}, \ldots \mathbf{s}_{M}} \sum_{m=1}^{M}\left\|\hat{\mathbf{r}}_{m}-\mathbf{A}_{2 m} \mathbf{s}_{m}-\sigma_{v}^{2} \mathbf{I}_{p, v}\right\|_{2}^{2} \\
\quad+\lambda \Gamma\left(\mathbf{s}_{1}, \ldots, \mathbf{s}_{M}\right) \\
\text { s.t. } s_{m l} \geq 0 \text { for } m=1, \ldots, M l=1, \ldots, L
\end{aligned}
$$

where $I_{P, v}=\operatorname{vec}\left(I_{P}\right)$ and $I_{P}$ is the unit matrix of dimension $P$.

It is obvious that (9) represents a group Lasso problem. Thus, we can reformulate the problem using the alternative representation:

$$
\begin{aligned}
& \underset{\mathbf{s}_{1}, \ldots \mathbf{s}_{M}}{\min } \Gamma\left(\mathbf{s}_{1}, \ldots, \mathbf{s}_{M}\right) \\
& \text { s.t. } \\
& \sum_{m=1}^{M}\left\|\hat{\mathbf{r}}_{m}-\mathbf{A}_{2 m} \mathbf{s}_{m}-\sigma_{v}^{2} \mathbf{I}_{P, v}\right\|_{2}^{2} \leq U \\
& \quad s_{m l} \geq 0 \text { for } m=1, \ldots, M, l=1, \ldots, L
\end{aligned}
$$

In order for (10) to be well defined, we need the value of $U$ to be dependent on the variance of the term $\sum_{m=1}^{M}\left\|\hat{\mathbf{r}}_{m}-\mathbf{A}_{\mathbf{2}_{m}} \mathbf{s}_{m}-\sigma_{v}^{2} \mathbf{I}_{P, v}\right\|_{2}^{2}$. By using the alternative representation (10) we switch the regularization parameter to a new value that is easier to calculate. From (4), in a similar fashion as (5), we have

so (10) can be rewritten as:

$$
\mathbf{r}_{m}=\operatorname{vec}\left(\mathbf{R}_{m}\right)=\mathbf{A}_{2 m} \mathbf{s}_{m}+\sigma_{v}^{2} \mathbf{I}_{P, v}
$$

$$
\begin{aligned}
& \underset{\mathbf{s}_{1}, \ldots \mathbf{s}_{M}}{\min } \Gamma\left(\mathbf{s}_{1}, \ldots, \mathbf{s}_{M}\right) \\
& \text { s.t. } \sum_{m=1}^{M}\left\|\hat{\mathbf{r}}_{m}-\mathbf{r}_{m}\right\|_{2}^{2} \leq U \\
& s_{m l} \geq 0 \text { for } m=1, \ldots, M, l=1, \ldots, L
\end{aligned}
$$

It has been shown in the COMET framework [20] that the covariance matrix $\mathbf{W}_{m}$ of $\hat{\mathbf{r}}_{m}-\mathbf{r}_{m}$ is:

$$
\mathbf{W}_{m}=E\left[\left(\hat{\mathbf{r}}_{m}-\mathbf{r}_{m}\right)\left(\hat{\mathbf{r}}_{m}-\mathbf{r}_{m}\right)^{H}\right]=\frac{1}{N}\left(\mathbf{R}_{m}^{T} \otimes \mathbf{R}_{m}\right)
$$

where $(\otimes)$ is the Kronecker product. Since $\hat{\mathbf{r}}_{m}-\mathbf{r}_{m}$ has correlated entries, it is very difficult to predict the value of $U$ in (11). But using the estimate of the decorrelation matrix $\quad \hat{\mathbf{W}}_{m}^{-1 / 2}=\sqrt{N}\left(\hat{\mathbf{R}}_{m}^{-T / 2} \otimes \hat{\mathbf{R}}_{m}^{-1 / 2}\right)$ as in [9], [20], we obtain an asymptotically normal distribution

$$
\hat{\mathbf{W}}_{m}^{-1 / 2}\left(\hat{\mathbf{r}}_{m}-\mathbf{r}_{m}\right) \sim \mathrm{N}\left(\mathbf{0}, \mathbf{I}_{P^{2}}\right)
$$

This asymptotic distribution allows us to reformulate (11) and find the regularization parameter:

$$
\begin{aligned}
& \min _{\mathbf{s}_{1}, \ldots \mathbf{s}_{M}} \Gamma\left(\mathbf{s}_{1}, \ldots, \mathbf{s}_{M}\right) \\
& \text { s.t. } \sum_{m=1}^{M}\left\|\hat{\mathbf{W}}_{m}^{-1 / 2}\left(\hat{\mathbf{r}}_{m}-\mathbf{r}_{m}\right)\right\|_{2}^{2} \leq c_{1}^{2} M P^{2} \\
& \quad s_{m l} \geq 0 \text { for } m=1, \ldots, M, l=1, \ldots, L
\end{aligned}
$$

where we have used the fact that when noise is uncorrelated the constraint should be set to a value close to the true variance (obtained from an infinite number of snapshots) of the noise - in our case $M P^{2}$. The constant $c_{1}$ is set to compensate for the estimation error of $\mathbf{W}_{m}^{-1 / 2}$ and the difference between the true and the estimated noise variance. Observing (13), the value of $c_{1}$ in (14) can be estimated based on the properties of the chi square distribution as in [9]. Namely the random variable $\sum_{m=1}^{M}\left\|\hat{\mathbf{W}}_{m}^{-1 / 2}\left(\hat{\mathbf{r}}_{m}-\mathbf{r}_{m}\right)\right\|_{2}^{2}$ is asymptotically chi square distributed and the probability $\eta$ that $\sum_{m=1}^{M}\left\|\hat{\mathbf{W}}_{m}^{-1 / 2}\left(\hat{\mathbf{r}}_{m}-\mathbf{r}_{m}\right)\right\|_{2}^{2} \geq c_{1}^{2} M P^{2}$ can be evaluated. The values $\eta$ and $c_{1}$ can be related through the Matlab function chi2inv as in [9], $c_{1}^{2} M P^{2}=\operatorname{chi} 2 \operatorname{inv}\left(1-\eta, M P^{2}\right)$.

Using the notation $\hat{\mathbf{r}}_{\mathbf{w} m}=\hat{\mathbf{W}}_{m}^{-1 / 2}\left(\hat{\mathbf{r}}_{m}-\sigma_{v}^{2} I_{P, v}\right)$, $\mathbf{A}_{\mathbf{3}_{m}}=\hat{\mathbf{W}}_{m}^{-1 / 2} \mathbf{A}_{\mathbf{2}_{m}}$ and introducing $\hat{\mathbf{r}}_{\mathbf{w}}=\left[\hat{\mathbf{r}}_{\mathbf{w} 1}^{T} \ldots \hat{\mathbf{r}}_{\mathbf{w} M}^{T}\right]^{T}$, $\mathbf{A}=\operatorname{diag}\left(\mathbf{A}_{31}, \ldots, \mathbf{A}_{\mathbf{3}_{M}}\right), \quad \mathbf{q}=\left[\mathbf{s}_{1}^{T} \ldots \mathbf{s}_{M}^{T}\right]^{T}=\left[q_{1} \ldots q_{L M}\right]^{T} \quad$ and $\Gamma_{1}(\mathbf{q})=\Gamma\left(\mathbf{s}_{1}, \ldots, \mathbf{s}_{M}\right)$ we obtain the following compact form:

$$
\begin{aligned}
\min _{\mathbf{q}} & \Gamma_{1}(\mathbf{q}) \\
\text { s.t. } & \left\|\hat{\mathbf{r}}_{\mathbf{w}}-\mathbf{A q}\right\|_{2} \leq c_{1} \sqrt{M} P \\
& q_{d} \geq 0 \text { for } d=1, \ldots, L M
\end{aligned}
$$

A form similar to (15) for the case when $M=1$ has been proposed in [23] but the authors do not consider the case when $M>1$ and do not make any connection to problem formulation (6) from [10] which is shown to be an efficient estimator for the narrowband $(M=1)$ scenario and was taken as a starting point for our wideband method.

After the optimization process, the directions of arrival are estimated by finding the directions that contain most of the power i.e. by calculating $G\left(\widetilde{\theta}_{l}\right)=\frac{1}{M} \sum_{z=0}^{M-1} q_{(l+L z)}$ and finding its peaks. The optimization problem (15) can be cast as a second order cone program:

$$
\begin{array}{ll}
\min _{\gamma_{l}, \mathbf{q}} \sum_{l=1}^{L} \gamma_{l} \\
\text { s.t. } \sqrt{\sum_{z=0}^{M-1} q_{(l+L z)}^{2}} \leq \gamma_{l} \text { for } l=1, \ldots, L \\
\left\|\hat{\mathbf{r}}_{\mathbf{w}}-\mathbf{A q}\right\|_{2} \leq c_{1} \sqrt{M} P \\
q_{d} \geq 0 \text { for } d=1, \ldots, L M
\end{array}
$$

The major component in the complexity of the method is the complexity of the optimization task (16). The time required to solve (16) depends on the number of elements 
in $\mathbf{q}$. To reduce this number we can use a simple approach of limiting the positions or elements in $\mathbf{q}$ included in the optimization. Namely, by preprocessing the received signal with some conventional technique we can roughly find the centers of the groups of DoAs and then distinguish between the individual DoAs using (15) with a reduced size vector $\mathbf{q}$ containing the directions in regions around the estimated centers. In this new reduced complexity algorithm, the number of elements in $\mathbf{q}$ will depend on the number of estimated centers and the size of the regions around group centers. A similar idea is used in [21], where, after the first stage and the estimation of the centers, additional modified linear transforms are used to improve the detection capabilities in the vicinity of the estimated centers.

\section{SimULATION RESULTS}

For performance evaluation of the proposed method, we compared it with the conventional methods: Minimum Variance Distortionless Response (MVDR) from [2] and MUltiple SIgnal Classification (MUSIC) modified for wideband signals from [3]. The simulation scenario considers the estimation of the direction of arrival for three uncorrelated signals arriving at the antenna array from $\left[90^{\circ} 95^{\circ} 165^{\circ}\right]$. The first two values are close enough to allow comparing the resolution capabilities of the methods. We assumed a wideband signal in the range between $800 \mathrm{MHz}$ and $1000 \mathrm{MHz}$ and obtained 30 uncorrelated narrowband signals from it, at equally spaced carrier frequencies starting from $f_{1}=800 \mathrm{MHz}$ with a frequency spacing $\Delta f=200 / 30 \mathrm{MHz}$ so that the narrowband requirement of bandwidth to center frequency ratio (fractional bandwidth) of less than 1\% [15], [16] is satisfied. We utilized every second narrowband signal, so that $M=15$, due to complexity reasons. We considered a circular antenna array with $P=8$ antennas and a radius equal to

$$
\rho=\frac{\frac{c}{f_{\max }}}{4 \sin \frac{\pi}{P}}
$$

which is the largest possible value not allowing spatial aliasing [22] ( $f_{\max }$ is the highest utilized frequency). We used [24] to solve the optimization problem (16). For the MUSIC algorithm we used linear transforms with $f_{0}$ equal to the median frequency of all $f_{m}$ and preliminary estimation of the DoA taken from the MVDR estimates using only the two directions of arrival with the highest power. The augmentation of the transform matrices was carried out using two separate approaches resulting in the algorithms "MUSIC1" and "MUSIC2". In "MUSIC1" we used random directions to augment the matrices for the linear transforms and in "MUSIC2" we used the Rotational Signal Subspace (RSS) method from [21] with additional angle components shifted from the estimated values for $\pm 0.125 \mathrm{BW}$ which was estimated to be $\pm 5^{\circ}$ for the utilized antenna array. The value for $B W$ was set to be equal to the Rayleigh resolution limit at frequency $f_{0}$ ([25], [3]). For all methods we assumed that the number of signals was known in advance. This can be achieved using one of the model selection techniques described in [1], [3] or [26]. For the proposed algorithm with reduced complexity termed "PropRed", we used the same preprocessing stage as for the MUSIC class algorithms i.e. the two strongest outputs from the MVDR algorithm. In accordance with the Rayleigh limit we chose the size of the regions around these DoAs at frequency $f_{0}$ to be $20^{\circ}$ on each side, i.e. around $0.5 \mathrm{BW}$ as recommended in [21]. We found that the restriction of the possible values in $\mathbf{q}$ could result in increased values for the end points, so the values $\gamma_{1}$ and $\gamma_{\max }$ in (16) were additionally restricted to be lower than 0.1 . The value $\gamma_{\max }$ corresponds to the last index in the $\gamma$ set used in the "PropRed" algorithm. The noise and each narrowband signal in every snapshot were generated as independent zero mean circular complex Gaussian random variables with variances equal to $\sigma_{v}^{2}=1$ and $P_{s}$, respectively. The signal to noise ratio was defined as $S N R=10 \log _{10} \frac{P_{s}}{\sigma_{v}^{2}}$. For estimating the covariance matrix of every narrowband signal $N=500$ snapshots were used in each trial. $L=180$ and $\widetilde{\theta}_{l} \in\{1,2, \ldots 180\}$ for all methods was used. Each point in the following figures was obtained by averaging the results from 1000 trials.

We start the comparison of methods by observing the average power spectra of all the methods at $\mathrm{SNR}=4 \mathrm{~dB}$, shown in Fig. 1. For the proposed method and the "PropRed" algorithm the average power spectrum is obtained by averaging $G\left(\widetilde{\theta}_{l}\right)$ over all trials. It is obvious that the proposed method and the "PropRed" algorithm, which are based on covariance fitting, on the average show very close approximation of the correct solution and the MVDR and MUSIC algorithms fail to distinguish the two different directions of arrival on the average. For the proposed method, we use $c_{1}=1.05$, that corresponds to $\eta=0.01$.

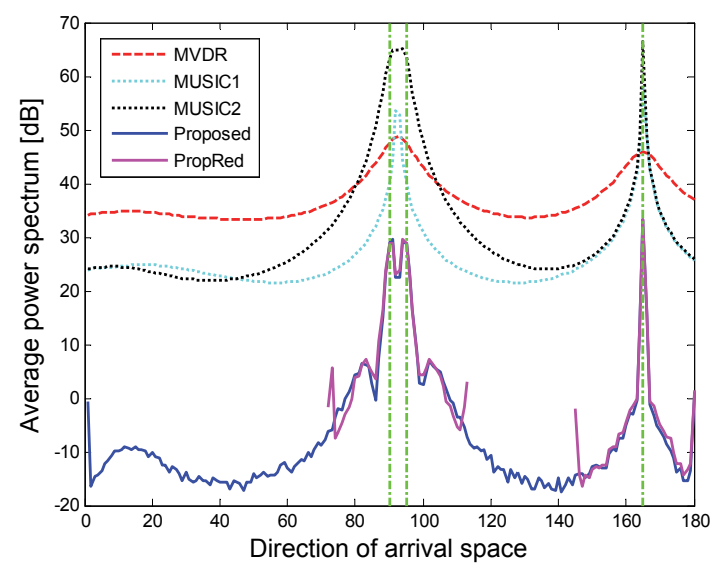

Fig. 1. The average spectra of three methods for $\mathrm{SNR}=4 \mathrm{~dB}$

Due to the utilization of the estimated version of covariance matrix $\mathbf{W}_{m}$ and the difference between the true and the estimated noise variance in (14), in some 
realizations additional spurious DoAs can be found in the spectrum of the proposed method, which can be seen in Fig. 1. This can be solved by using one of the known methods for estimation of the number of arriving signals [1], [3]. For the same reason in some realizations the optimization problem (15) is infeasible. In such cases we propose using the standard optimization $\min _{\mathbf{q}}\left\|\hat{\mathbf{r}}_{\mathbf{w}}-\mathbf{A q}\right\|_{2}$

s.t. $q_{d} \geq 0$ for $d=1, \ldots, L M$, which always converges, in order to get the estimates of the DoAs.

Next, we compare the three methods based on two criteria: the probability that the first three estimated values are the true values, and the number of estimated peaks in the power spectrum that are within $15 \mathrm{~dB}$ of the highest peak in that spectrum.

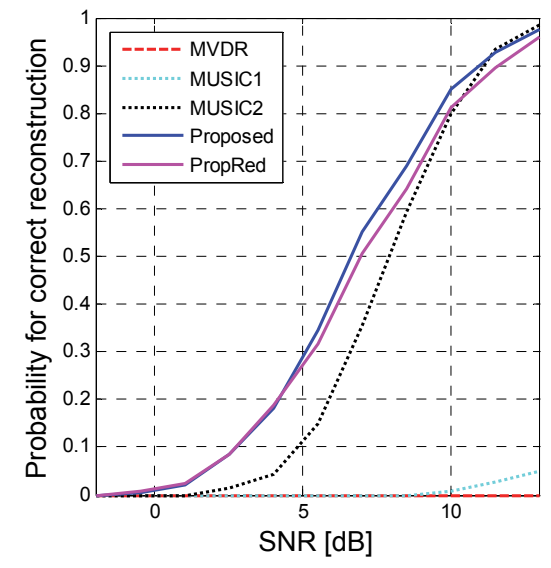

(a) Probability of correct reconstruction vs SNR.

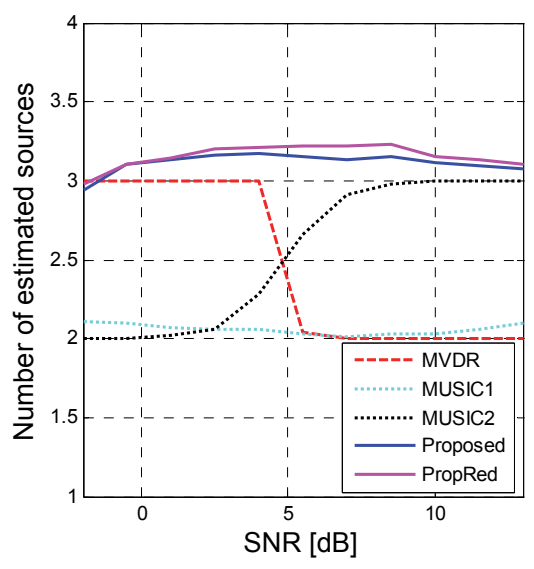

(b) Number of estimated sources vs SNR.

Fig. 2. Average performance of considered methods.

Fig. 2 (a) shows that the proposed method performs better compared to the other considered methods when correct directions are required. The performance improvement is present in the low and moderate correct reconstruction probability regions, but with the increase of correct reconstruction probability, the performance of "MUSIC2" algorithm becomes similar and even slightly better than the performance of the proposed one. The "PropRed" algorithm in the low correct reconstruction probability region shows a similar performance as the more complex version does but in the high probability region there is some performance loss. From Fig. 2 (a) it is obvious that for the methods based on coherent combining ("MUSIC1" and "MUSIC2") the good choice of the transform matrices is very important. This is not the case for the proposed method. On the other hand, Fig. 2 (b) shows that the capability of the proposed method for estimation of the number of directions of arrival needs some improvement, since some spurious peaks are present over the whole observed range of SNR values. We believe that the improvement can be achieved by an appropriate choice of the constant $c_{1}$ but this is out of the scope of this paper. Although the number of estimated directions for the "MVDR" method in the low SNR region corresponds to the correct number of directions, two of the estimated directions are close to the true positions and the third one is a false peak at around $15^{\circ}$ shown in Fig. 1. It should be noticed that the complexity of the proposed method is much higher than the complexity of the MVDR and MUSIC and the complexity gap increases with the increase of $M$. Simulations were carried out on a PC with Intel i3-2100 processor working at $3.1 \mathrm{GHz}$ and the required time for solving (16) was around 35.5 seconds. The required time for solving (16) for the reduced complexity algorithm was around 12.5 seconds which was proportional to the decrease of the number of elements in q. The additional reduction of the size of regions around the estimated DoAs, especially below $10^{\circ}$ on each side, leads to a significant performance loss. The time required for all operations included in the "MUSIC2" algorithm excluding the preprocessing was around $3.3 \mathrm{~ms}$.

In order to illustrate the gain from using multiple narrowband channels, in Fig 3 the probability of correct reconstruction is shown for different numbers of narrowband channels. For all simulations in this part, we used $\eta=0.01$. It should be noticed that we are assuming that the narrowband signals are mutually uncorrelated, which in practice can be incorrect if too many narrowband channels are created in a strictly defined region. This could be a topic of future research.

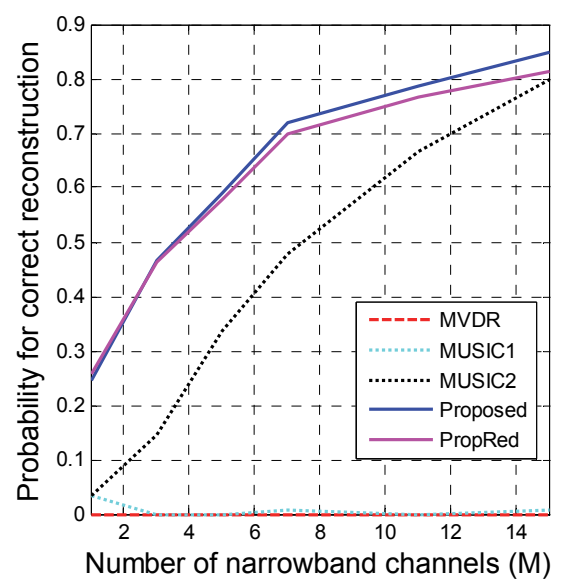

Fig. 3. Probability of correct reconstruction vs $M$ for $\mathrm{SNR}=10 \mathrm{~dB}$.

Fig. 3 shows that the increase of the number of utilized narrowband channels for the proposed algorithm leads to performance improvement. As in the previous case, the performance improvement for the transform based approaches is highly dependent on the transform matrices, while the proposed method does not have such issues. 


\section{CONCLUSION}

A new method for the direction of arrival estimation of wideband signals is proposed. The method is based on the existing narrowband covariance fitting method by additionally introducing group sparsity regularization and appropriately choosing the regularization constants for both the individual sparsity and the group sparsity. The method shows improved performance compared to the existing conventional methods for direction of arrival estimation of wideband signals based on MUSIC and MVDR in terms of the probability of correct reconstruction in the region of low to moderate probability, but needs additional estimation of the number of directions of arrival and has much higher complexity.

\section{APPENDIX}

In order to show the equivalence between (8) and (9) it is sufficient to show that $T_{m}=\left\|\hat{\mathbf{r}}_{m}-\mathbf{A}_{2 m} \mathbf{s}_{m}-\sigma_{v}^{2} \mathbf{I}_{P, v}\right\|_{2}^{2}$ can be represented as $\left\|\hat{\mathbf{r}}_{m}-\mathbf{A}_{2 m} \mathbf{s}_{m}\right\|_{2}^{2}+2 P \sigma_{v}^{2} \mathbf{1}^{T} \mathbf{s}_{m}+C$, where $C$ is a constant independent of $\mathbf{s}_{m}$ :

$$
\begin{aligned}
T_{m} & =\left\|\hat{\mathbf{r}}_{m}-\mathbf{A}_{2 m} \mathbf{s}_{m}-\sigma_{v}^{2} \mathbf{I}_{P, v}\right\|_{2}^{2} \\
& =\left(\hat{\mathbf{r}}_{m}^{H}-\mathbf{s}_{m}^{T} \mathbf{A}_{2 m}{ }^{H}-\sigma_{v}^{2} \mathbf{I}_{P, v}^{T}\right)\left(\hat{\mathbf{r}}_{m}-\mathbf{A}_{2 m} \mathbf{s}_{m}-\sigma_{v}^{2} \mathbf{I}_{P, v}\right) \\
& =\left\|\hat{\mathbf{r}}_{m}-\mathbf{A}_{2 m} \mathbf{s}_{m}\right\|_{2}^{2}-\left(\hat{\mathbf{r}}_{m}^{H}-\mathbf{s}_{m}^{T} \mathbf{A}_{2 m}{ }^{H}\right) \mathbf{I}_{P, v} \sigma_{v}^{2} \\
& -\sigma_{v}^{2} \mathbf{I}_{P, v}^{T}\left(\hat{\mathbf{r}}_{m}-\mathbf{A}_{2 m} \mathbf{s}_{m}\right)+\sigma_{v}^{2} \mathbf{I}_{P, v}^{T} \mathbf{I}_{P, v} \sigma_{v}^{2} \\
& =\left\|\hat{\mathbf{r}}_{m}-\mathbf{A}_{2 m} \mathbf{s}_{m}\right\|_{2}^{2}+\mathbf{s}_{m}^{T} \mathbf{A}_{2 m}^{H} \mathbf{I}_{P, v} \sigma_{v}^{2} \\
& +\sigma_{v}^{2} \mathbf{I}_{P, v}^{T} \mathbf{A}_{2 m} \mathbf{s}_{m}+C
\end{aligned}
$$

where $\quad C=-\sigma_{v}^{2} \mathbf{I}_{P, v}^{T} \hat{\mathbf{r}}_{m}-\hat{\mathbf{r}}_{m}^{H} \mathbf{I}_{P, v} \sigma_{v}^{2}+\sigma_{v}^{2} \mathbf{I}_{P, v}^{T} \mathbf{I}_{P, v} \sigma_{v}^{2} \quad$ is $\quad$ a

constant independent of $\mathbf{s}_{m}$. To complete the proof, we have to show that $\mathbf{s}_{m}^{T} \mathbf{A}_{2 m}{ }_{2}^{H} \mathbf{I}_{P, v}=P \mathbf{1}^{T} \mathbf{s}_{m}$ and $\mathbf{I}_{P, v}^{T} \mathbf{A}_{2 m} \mathbf{s}_{m}=$ $P \mathbf{1}^{T} \mathbf{s}_{m}$. In order to do so we should return to the definition of $\mathbf{A}_{2 m}$. Namely, the columns of $\mathbf{A}_{2 m}$ are obtained by vectorization of the matrices $\mathbf{a}\left(\tilde{\theta}_{l}, f_{m}\right) \mathbf{a}\left(\tilde{\theta}_{l}, f_{m}\right)^{H}$ that have diagonal elements equal to 1 , the power gain of the antennas. Additionally, $\mathbf{I}_{P, v}$ is obtained by vectorization of the unit matrix so the product between $\mathbf{I}_{P, v}^{T}$ and any column of $\mathbf{A}_{2 m}$ is equal to the sum of the diagonal elements of the matrix $\mathbf{a}\left(\tilde{\theta}_{l}, f_{m}\right) \mathbf{a}\left(\tilde{\theta}_{l}, f_{m}\right)^{H}$ which is equal to $P$, the number of elements in the antenna array. Thus, $\mathbf{I}_{P, v}^{T} \mathbf{A}_{\mathbf{2}_{m}}=P \mathbf{1}^{T}$ and $\mathbf{I}_{P, v}^{T} \mathbf{A}_{\mathbf{2}_{m}} \mathbf{s}_{m}=P \mathbf{1}^{T} \mathbf{s}_{m}$. Since $\mathbf{s}_{m}^{T} \mathbf{A}_{\mathbf{2}_{m}} \mathbf{I}_{P, v}$ is a real scalar, then $\mathbf{s}_{m}^{T} \mathbf{A}_{\mathbf{2}_{m}}{ }^{H} \mathbf{I}_{P, v}=\left(\mathbf{s}_{m}^{T} \mathbf{A}_{\mathbf{2}_{m}}{ }^{H} \mathbf{I}_{P, v}\right)^{H}=\mathbf{I}_{P, v}^{T} \mathbf{A}_{\mathbf{2} m} \mathbf{s}_{m}$ and, thus, $\mathbf{s}_{m}^{T} \mathbf{A}_{\mathbf{2}_{m}}^{H} \mathbf{I}_{P, v}=P \mathbf{1}^{T} \mathbf{s}_{m}$.

\section{REFERENCES}

[1] L. Godara, "Applications of antenna arrays to mobile communications. I. performance improvement, feasibility, and system considerations," Proceedings of the IEEE, vol. 85, no. 7, pp. 1031-1060, july 1997

[2] J. Krolik and D. Swingler, "Multiple broad-band source location using steered covariance matrices," IEEE Trans. on Acoustics, Speech and Signal Processing, vol. 37, no. 10, pp. 1481-1494, 1989.
[3] H. Wang and M. Kaveh, "Coherent signal-subspace processing for the detection and estimation of angles of arrival of multiple wideband sources," IEEE Transactions on Acoustics, Speech and Signal Processing, vol. 33, no. 4, pp. 823-831, 1985.

[4] D. L. Donoho, "Compressed sensing," IEEE Transactions on Information Theory, vol. 52, no. 4, pp. 1289-1306, 2006.

[5] D. Malioutov, M. Cetin, and A. Willsky, "A sparse signal reconstruction perspective for source localization with sensor arrays," IEEE Transactions on Signal Processing, vol. 53, no. 8, pp. 3010-3022, 2005

[6] M. Hyder and K. Mahata, "Direction-of-arrival estimation using a mixed $l_{2,0}$ norm approximation," IEEE Transactions on Signal Processing, vol. 58, no. 9, pp. 4646-4655, 2010.

[7] X. Xu, X. Wei, and Z. Ye, "DOA estimation based on sparse signal recovery utilizing weighted $l_{1}$-norm penalty," IEEE Signal Processing Letters, vol. 19, no. 3, pp. 155-158, 2012.

[8] Z.-M. Liu, Z.-T. Huang, and Y.-Y. Zhou, "Direction-of-arrival estimation of wideband signals via covariance matrix sparse representation," IEEE Trans. on Signal Processing, vol. 59, no. 9, pp. 4256-4270, sept. 2011

[9] J. Yin and T. Chen, "Direction-of-arrival estimation using a sparse representation of array covariance vectors," IEEE Transactions on Signal Processing, vol. 59, no. 9, pp. 4489-4493, 2011.

[10] J. Zheng and M. Kaveh, "Sparse spatial spectral estimation: A covariance fitting algorithm, performance and regularization," IEEE Trans. on Signal Processing, vol. 61, no. 11, pp. 2767-2777, 2013.

[11] L. Blanco and M. Najar, "Sparse covariance fitting for direction of arrival estimation," EURASIP Jour. on Adv. in Sig. Proc., pp. 1-11, 2012.

[12] P. Stoica, P. Babu, and J. Li, "SPICE: A sparse covariance-based estimation method for array processing," IEEE Transactions on Signal Processing, vol. 59, no. 2, pp. 629-638, 2011.

[13] S. Pejoski and V. Kafedziski, "Indirect method for direction of arrival estimation of bandlimited communication signal based on compressed sensing," in IEEE EUROCON, July 2013, pp. 584-590.

[14] B. Miller, J. Goodman, K. Forsythe, J. Sun, and V. Goyal, "A multisensor compressed sensing receiver: Performance bounds and simulated results," in Conf. Record of the Forty-Third Asilomar Conf on Signals, Systems and Comp., nov. 2009, pp. 1571-1575.

[15] Y. Wang, G. Leus, and A. Pandharipande, "Direction estimation using compressive sampling array processing," in Proc. of IEEE/SP 15th Workshop on Stat. Sig. Proc., sept. 2009, pp. 626-629.

[16] M. Ghavami, "Wideband smart antenna theory using rectangular array structures," IEEE Transactions on Signal Processing, vol.50, no.9, pp.2143,2151, Sep 2002

[17] T. Do-Hong, P.Russer, "Signal processing for wideband smart antenna array applications," Microwave Magazine, IEEE, vol.5, no.1, pp.57,67, Mar 2004

[18] P. Sprechmann, I. Ramirez, G. Sapiro, and Y. Eldar, "C-HiLasso: A collaborative hierarchical sparse modeling framework," IEEE Transactions on Signal Processing, vol. 59, no. 9, pp. 4183-4198, 2011.

[19] E. Dall'Anese, J. A. Bazerque, and G. B. Giannakis, "Group sparse Lasso for cognitive network sensing robust to model uncertainties and outliers," Physical Comm.., vol. 5, no. 2, pp. 161 - 172, 2012.

[20] P. Ottersten, B Stoica and R. Roy, "Covariance matching estimation techniques for array signal processing applications," Digital Signal Processing, vol. 8, no. 3, pp. 185-210, 1998.

[21] H. Hung and M. Kaveh, "Focussing matrices for coherent signalsubspace processing," IEEE Transactions on Acoustics, Speech and Signal Processing, vol. 36, no. 8, pp. 1272-1281, Aug 1988.

[22] J. Dmochowski, J. Benesty, and S. Affes, "Direction of arrival estimation using the parameterized spatial correlation matrix," IEEE Transactions on Audio, Speech, and Language Processing, vol. 15, no. 4, pp. 1327-1339, May 2007.

[23] N. Hu, Z. Ye, X. Xu, and M. Bao, "DOA estimation for sparse array via sparse signal reconstruction," IEEE Trans. on Aerospace and Electronic Systems, vol. 49, no. 2, pp. 760-773, april 2013.

[24] M. Grant and S. Boyd, "CVX: Matlab software for disciplined convex programming, version 2.0 beta," http://cvxr.com/cvx, 2013.

[25] H. L. Van Trees, Optimum Array Processing (Detection, Estimation, and Modulation Theory, Part IV), 1st ed. John Wiley \& Sons, Inc., Mar. 2002.

[26] A. de Brauwere, F. De Ridder, R. Pintelon, M. Elskens, J. Schoukens, and W. Baeyens, "Model selection through a statistical analysis of the minimum of a weighted least squares cost function," Chemometrics and Intelligent Laboratory Systems, vol. 76, no. 2, pp. 163-173, 2005. 